\title{
Optic nerve glioma and the management of optic nerve tumours in the young
}

\author{
J E WRIGHT, A A McNAB, AND W I McDONALD \\ From the Orbital Clinic, Moorfields Eye Hospital, City Road, London EC1V 2PD
}

SUMMARY Thirty-one patients presenting as orbital optic nerve glioma have been reviewed with maximum follow-up of 14 years. Sixteen of these patients have been reported on previously and further follow-up is provided. Sixteen patients had a stable clinical course with little change over a period of up to 13.5 years. Neurofibromatosis was relatively common in this group (11/16). Fifteen patients had progressive enlargement of the tumour; the incidence of neurofibromatosis in this group was low (4/15). Eleven of these patients were explored neurosurgically and the optic nerve totally excised in 10 of them. The proximal cut end was normal in six patients and the chiasm has apparently remained free of tumour in all of them. We suggest a method of management of primary optic nerve tumours, both meningiomas and gliomas, in young patients.

There is continuing debate about the natural history and management of optic nerve gliomas. Hoyt and Baghdassarian' and others ${ }^{2-4}$ have claimed that these tumours behave as hamartomas and that they should be managed conservatively. Others have reached different conclusions, stating that these tumours are not benign and self-limiting, and that treatment is required..$^{5-7}$ In 1980 we reported on 17 patients and demonstrated a division into one group which was stable and another which showed active growth. ${ }^{5}$ One of these patients was subsequently found to have a meningioma, not a glioma as was first reported, and is described as case 1 in the accompanying paper. ${ }^{8}$ Since then we have seen a further 15 patients and they too have fallen into one of these two groups. We have adopted a radical surgical approach in those patients with evidence of active growth and without evidence of chiasmal involvement. In this paper we report our results and follow-up of 31 patients with this uncommon condition.

\section{Patients and methods}

Since 1973 we have seen 31 patients with an initial diagnosis of optic nerve glioma. The diagnosis was made on the basis of typical clinical and radiological findings, and in 16 patients in whom diagnostic tissue

Correspondence to J E Wright, FRCS, Moorfields Eye Hospital, City Road, London EC1V 2PD. was obtained our clinical diagnosis was confirmed histopathologically.

We have excluded the small number of older adults with the highly malignant form of optic glioma (glioblastoma), which usually involves the intracranial visual pathways and follows a quite different course. ${ }^{9}$

A detailed clinical and family history was obtained and a full ocular and neurological examination performed in each patient. Visual fields were charted whenever possible, and most patients had pattern visual evoked potentials (VEP) recorded. Patients were carefully examined for stigmata of von Recklinghausen's neurofibromatosis by the criteria of Crowe and Schull." Plain skull films with optic canal views were routinely performed, and since 1975 all patients have had CT scans at presentation. In recent years most patients have also undergone magnetic resonance imaging (MRI).

The patients were assigned to two groups, (1) those with a 'stable' course and (2) those with 'active growth', on the basis of changes in vision and/or proptosis. A change was indicated by either the history (patient's or relatives' observations) or follow-up examination. For inclusion in the stable group our criteria were: (a) unchanged or improved vision, or vision worsening by less than 2 lines over a minimum period of two years, and (b) proptosis unchanged, decreasing or increasing by $2 \mathrm{~mm}$ or less over a minimum of two years. For inclusion in the 
active growth group the criteria were $(a)$ visual loss at the rate of more than 2 lines in a year, or $(b)$ a rate of increase in the proptosis of more than $2 \mathrm{~mm}$ in a year. This leaves a potential gap for 'intermediate' patients. None of our patients qualified for this category.

While the patients had a spectrum of change in vision and/or acuity, they fell clearly into one or other group. For example, patients with active growth usually had a dramatic increase of proptosis over periods of less than a year, whereas those in the stable group remained unchanged or even improved over many years.

Follow-up was every six to 12 months in the group with an established stable course but every two to six months in the group with evidence of active growth.

Clinical, radiological, and electrophysiological investigations were aimed at determining the anatomical extent of the tumour and in particular whether the optic chiasm was involved. Those patients with active growth, poor vision, and no evidence of chiasmal disease were referred to a neurosurgeon for a frontal craniotomy. If the chiasm appeared macroscopically normal, the nerve was transected just in front of the chiasm, and after unroofing the optic canal and orbital roof the involved nerve was removed to the globe.

In patients in whom there was active growth but investigations revealed an involved chiasm surgery, when required, was confined to the reduction of the disfiguring proptosis by means of an anterior approach, or a ventriculo-atrial shunt for obstructive hydrocephalus.

Biopsy of the optic nerve has not been performed for many years because the results can be misleading. ${ }^{5}$ Radiotherapy was not used routinely, and only two patients have had this form of treatment. Squint surgery was occasionally performed for cosmetic purposes and enucleation was required in several cases.

\section{Results}

\section{CLINICAL FINDINGS}

A total of 31 patients has been seen with a clinical diagnosis of optic nerve glioma. The details of their age and sex distribution are shown in Table 1. The presenting symptoms are outlined in Table 2 and the physical findings in Table 3. Fifteen of the 31 patients had neurofibromatosis (48\%). All had reduced acuity at presentation, with 15 having $6 / 60$ or worse vision (Table 4 ). the average degree of proptosis was $3 \cdot 0$ $\mathrm{mm}$, but six had no measurable proptosis whereas one was proptosed $8 \mathrm{~mm}$. All but one patient had a relative afferent pupil defect, and this patient was found to have bilateral optic nerve involvement on CT but had presented with unilateral proptosis and visual failure. The ocular motility disturbance most commonly seen (14/31) was a limitation of elevation of the globe, which in many cases was quite marked.

\section{RADIOLOGY}

An enlarged optic foramen on plain $x$-ray was found in 25 patients at presentation. Twenty-six patients had CT scans at presentation (Fig. 1); 24 showed a mass involving the optic nerve without apparent involvement of the optic chiasm. Two showed obvious chiasmal enlargement and two incidental enlargement of the contralateral optic nerve. These changes were unsuspected on the basis of clinical examination.

Magnetic resonance imaging (MRI) has been

Table 1 Age and sex data

\begin{tabular}{lll}
\hline Total patients & & 31 \\
Sex & Male 7 & Female 24 \\
Involved nerve & Right 15 & Left 16 \\
Age at presentation & Mean & $10 \cdot 2$ years \\
& Median & $6 \cdot 5$ years \\
& Range & $2-38$ years \\
\hline
\end{tabular}

Table 2 Presenting symptoms

\begin{tabular}{lc}
\hline Proptosis only & 12 \\
Proptosis and visual loss & 9 \\
Visual loss only & 3 \\
Proptosis and squint & 3 \\
Squint and visual loss & 2 \\
Squint only & 1 \\
Pain, visual loss, and obscurations on & 1 \\
$\quad$ lateral gaze & \\
\hline
\end{tabular}

Table 3 Physical findings

\begin{tabular}{llc}
\hline & Present & Absent \\
\hline Poor vision & 31 & - \\
Proptosis & 25 & 6 \\
Relative afferent pupil defect & 30 & 1 \\
Enlarged optic canal & 24 & 7 \\
Neurofibromatosis & 15 & 16 \\
\hline
\end{tabular}

Table 4 Visual acuity

\begin{tabular}{ll}
\hline Vision & Number \\
\hline $6 / 9-6 / 12$ & 5 \\
$6 / 18-6 / 24$ & 6 \\
$6 / 36-6 / 60$ & 9 \\
CF-HM & 4 \\
PL & 3 \\
NPL & 4 \\
\hline
\end{tabular}

$\mathrm{CF}=$ counting fingers. $\mathrm{HM}=$ hand movements. $\mathrm{PL}=$ perception of light. $\mathrm{NPL}=$ no $\mathrm{PL}$. 


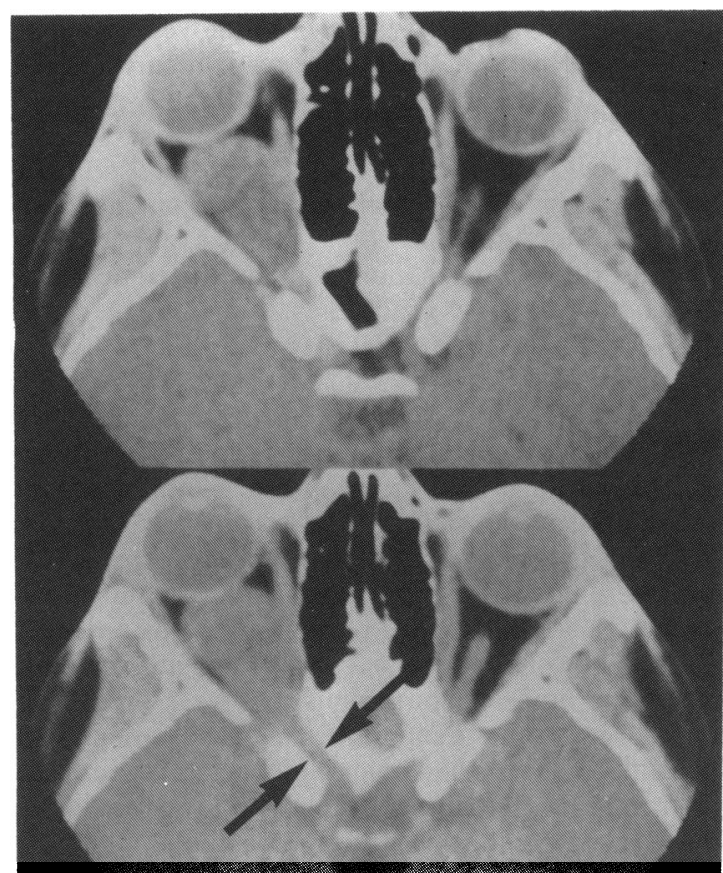

Fig. 1 Axial CT, without contrast, of a patient with an active glioma involving the right optic nerve. Note the typical fusiform outline of the tumour and the enlarged optic canal (arrowed).

particularly useful for demonstrating the extent of tumour (Figs. 2 and 3). In two patients it has detected chiasmal involvement when both CT and VEPs were normal.

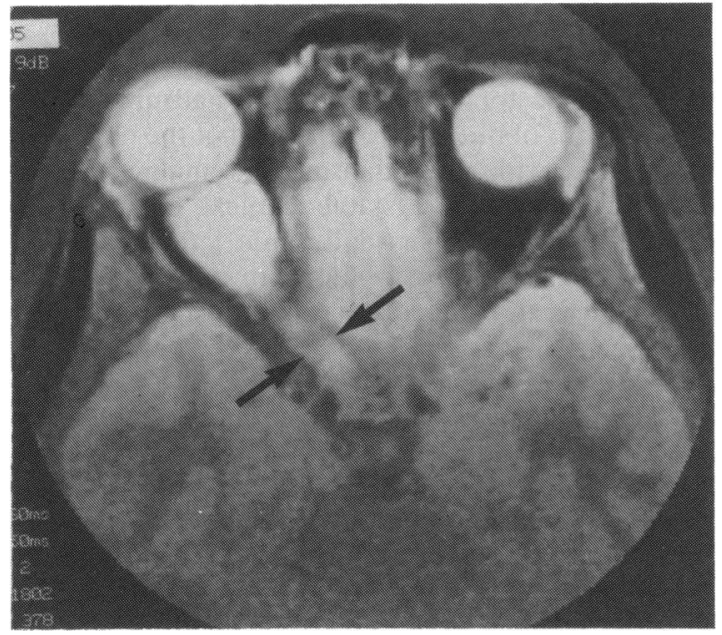

Fig. 2 Axial MRI of the same patient as in Fig. 1. Note the improved definition of the enlarged optic nerve in the optic canal (arrowed).

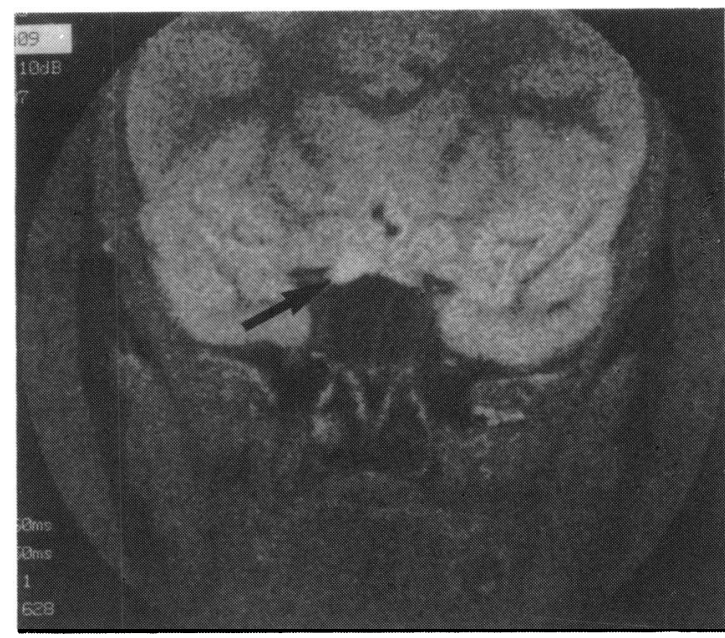

Fig. 3 Coronal MRI of the same patient in Figs. 1 and 2 showing involvement of the optic chiasm (arrowed).

CLINICAL COURSE

Stable group

Eight of nine patients reported in $1980^{5}$ as having a stable clinical course have not shown evidence of active growth subsequently. One patient has since been found to have a meningioma and has been excluded from this study (patient 1 in the accompanying paper on optic nerve meningioma ${ }^{\mathrm{x}}$ ). Eight new patients have been added to this group. The stable group had ages at presentation ranging from 2.2 to 38 years (mean 9.9 years, median 8 years), and at the onset of their symptoms from birth to 30 years (mean 5.9 , median 3.5 years). Eleven of 16 had neurofibromatosis. In three patients in whom clinical examination suggested the glioma was confined to one optic nerve CT scans showed thickening of the contralateral nerve in two and the chiasm in the other. The optic disc was atrophic in 14/16 patients, with shunt vessels in one. Only two showed optic disc swelling, which was mild. Visual acuity improved spontaneously in three patients, one from 6/24 to $6 / 6$ over seven years, and minor fluctuations in vision were common. Two patients also had a reduction in their proptosis of 2 and $3 \mathrm{~mm}$ in the period of followup. The greatest increase in proptosis was from 3 to 6 $\mathrm{mm}$ over six years. The remainder were unchanged or had increases of 1 or $2 \mathrm{~mm}$ over periods of two to 12 years. Follow-up has ranged from two to 13.5 years (mean $7 \cdot 2$, median $7 \cdot 0$ years). The exception was a patient with a one-month follow-up who presented at the age of 38 with a 27-year history of poor but stable vision and neurofibromatosis. Four patients within this group have been lost to follow-up after 2, 2.5, 3, and 8 years. Another patient within 
this group with neurofibromatosis and a five-year follow-up has a mother (not included in this series) with a chiasmal glioma that was irradiated and has remained essentially unchanged over at least 10 years.

\section{Active group}

By contrast there were 15 patients with evidence of progressive enlargement of the tumour, eight added since 1980.5 These patients presented between the ages of 3.0 and 26.4 years (mean 10.7, median 9.0 years), and their symptoms began between birth and $26 \cdot 0$ years (mean $8 \cdot 3$, median 6.9 years). Only four of this group had neurofibromatosis. Nine had disc swelling, four optic atrophy, one had tumour on the disc, and another a total exudative retinal detachment.

Two patients from the 'active growth' group were confirmed as having chiasmal involvement by CT. One presented in 1974 ('patient 3') with a fourmonths history of squint and two months' proptosis. During follow-up she developed visual evoked potential (VEP) evidence, and later visual field changes, indicative of chiasmal involvement. A CT in 1974 had failed to show chiasmal involvement but a later higher resolution scan detected it. The second patient presented first in 1973, ('patient 7's) and during follow-up developed VEP and then perimetric evidence of chiasmal involvement. Only at this stage did a CT scan disclose an enlarged chiasm, and a pneumoencephalogram confirmed a suprasellar mass. A third patient's chiasmal disease was detected only on MRI.

Follow-up in the 'active growth' group has been from 1.5 to 14 years (mean $7 \cdot 8$, median 6.2 years). No patient has been lost to follow-up. One died after a pneumoencephalogram.

\section{SURGERY}

Ten patients with active growth without evidence of chiasmal disease were referred for neurosurgical exploration. Nine had a macroscopically normal chiasm and the optic nerve was therefore excised from the chiasm to the globe. The proximal end of the excised nerve was histologically clear of tumour in six patients and involved by tumour in three patients. One patient had an enlarged chiasm and the tumour was merely biopsied. A further patient had an orbital glioma excised through a lateral orbitotomy. Whether the tumour extended into the intracanalicular portion of the nerve is not known.

Three patients required enucleation. One had tumour growth into the vitreous and developed intractable glaucoma. One had massive growth of glioma from a small amount of tumour which was left attached to the globe after the initial craniotomy. The globe and recurrent glioma were removed together. The third patient had multiple cystic recurrences of the orbital part of the glioma and ultimately required enucleation and marsupialisation of the cyst. Several patients who have had the optic nerve excised have developed signs of ocular ischaemia, but all have retained the globe. The one patient explored via a lateral orbitotomy ('patient 8 ') had the optic nerve containing a large grade 1 astrocytoma excised. There has been no evidence of recurrence 12 years after surgery.

Two patients with active growth and chiasmal involvement needed debulking of the orbital part of the glioma for disfiguring proptosis and intermittent subluxation of the globe.

In the group with a stable course five have had surgery. In two a biopsy was obtained to confirm the diagnosis. We have since discontinued this practice, as small biopsies tend to give misleading information, and large biopsies compromise vision. Three have undergone cosmetic squint surgery. One patient with neurofibromatosis had a large plexiform neuroma in association with a diffusely enlarged ipsilateral optic nerve. The plexiform neuroma was debulked. The combination of a plexiform neuroma and a presumed optic nerve glioma is in our experience very unusual.

\section{HISTOPATHOLOGY}

In 14 of the 15 cases with evidence of active growth pathological tissue was obtained for histological examination. Two specimens were obtained at orbitotomy to debulk tumour in patients with known chiasmal disease. Eleven specimens were excised optic nerves, and one was a biopsy obtained at craniotomy. In $11 / 14$ a typical grade 1 pilocytic astrocyoma was found. Arachnoid hyperplasia was prominent in seven. Three were considered to be grade 2 astrocytomas. These three all had microscopic involvement of the cut proximal end of the nerve but any disease within the chiasm has remained stable. Two patients with a stable clinical course were biopsied via orbitotomy and grade 1 tumours were found. There was no relationship between neurofibromatosis and the pattern or grade of glioma.

\section{FURTHER CLINICAL COURSE}

Of the three patients in whom tumour was present in the proximal end of their excised optic nerve, only one has shown evidence of advancement of the residual intracranial tumour, with the development of mild disc swelling, an inferonasal field defect, an abnormal VEP and involvement of the chiasm and the contralateral optic nerve visible with MRI (but not CT). These changes have remained stable over five years, and central vision remains $6 / 6$ seven years after craniotomy. This patient also developed an 
orbital recurrence from tumour left attached to the globe and required an enucleation. No further orbital recurrence has occurred. In the two other patients there has been no clinical or radiological evidence of tumour growth within the chiasm over periods of six and nine years, but one of these patients developed multiple cystic orbital recurrences and finally required enucleation.

None of the six patients in whom the chiasm was clear after excision of the optic nerve has evidence of recurrent chiasmal disease after being followed up for $2,3,5,9,10$, and 10 years. One developed an orbital recurrence requiring enucleation and then partial exenteration for a further recurrence. The orbit is free of tumour after five years. One patient whose glioma was removed via a lateral orbitotomy has had no chiasmal involvement after 12 years. We do not know if the canalicular part of the optic nerve is free of tumour.

\section{Discussion}

The natural history and management of optic nerve gliomas remains controversial. Some authors ${ }^{1-3}$ believe them to behave like hamartomas which are incapable of extension along the nerve into the chiasm and therefore offer no threat to the vision of the other eye. Worsening vision or involvement of the contralateral field is attributed to an increase in the pressure of a pre-existing lesion on near-by axons rather than evidence of true extension into previously uninvolved tissue. We disagree with this concept. The evidence from our present study supports the views we expressed in $1980 . .^{5}$ At that time we concluded that patients with optic nerve glioma fell into two fairly distinct groups, one with an indolent course and little change in the size of the tumour, and the other with progressive tumour enlargement. Having followed up these patients for a longer period and added new patients to the series, our conclusions remain the same. Two papers have recently added support for this view. Alvord and Lofton ${ }^{7}$ reviewed the literature and applied actuarial analysis to 623 cases of optic gliomas of which 155 occurred in the optic nerve. They concluded that gliomas have a very wide but continous range of growth rates; some grow rapidly probably by simple exponential doubling at a constant rate, but the majority behave as though growth decelerates. Wong $e t$ al $^{\circ}$ have published a reanalysis of the University of California, San Francisco, experience. Several of their patients had been the subject of Hoyt's studies, ' but they reached a different conclusion, namely, that optic gliomas are not benign, self-limiting lesions and should be treated.

Deciding whether patients had an inactive or active lesion was usually straightforward. Most patients had an initial period of growth of the lesion with progressive proptosis. A minority of those with an inactive lesion had little or no proptosis at any stage, and poor vision was noted either accidentally or at routine school examination. The majority progressed to a period of quiescence, which we have observed to last a long as 13 years, with one patient reporting an interval of 27 years. Some while under observation experienced a reduction of proptosis and improved visual acuity. Active progressive gliomas continued to enlarge, with worsening vision and increasing proptosis usually accompanied by swelling of the optic disc and CT or MRI evidence of growth of the tumour.

The frequency of disc swelling and restriction of ocular movement in patients with active lesions is probably due to enlargement of the tumour. Nine patients had disc oedema compared with only two in the inactive group, where optic atrophy was much more common (14/16). Similarly, restriction of movement of the eye, usually limitation of elevation, was present in 11 patients with active growth, whereas only three patients with a stable course had any reduction of movement of the globe.

The most striking difference between the two groups was the higher incidence of neurofibromatosis in patients with a stable course (11/16) compared with those with active growth (4/15). The tendency for gliomas of the optic pathways, when associated with neurofibromatosis, to behave in an indolent manner has been noted previously. ${ }^{11-15}$ The overall incidence of neurofibromatosis in our patients was $48 \%$. This is comparable to that observed in some other reports, ${ }^{12}{ }^{13}$ but rates in other series have been lower $(10-30 \%) .^{415} 16$

We have shown in this series that, while half of the optic nerve gliomas remained quiescent, the remainder enlarged. The crucial question is whether this enlargement is due to division of tumour astrocytes with spread along the optic nerve or is merely an increase in bulk caused by the accumulation of mucin or an associated desmoplastic reaction in the meninges. While the increase in the bulk of the tumour can be easily demonstrated with CT or even better with MRI, ${ }^{17-19}$ it is extremely difficult to define the extent of the astrocytoma. The histopathologist examining appropriately stained sections of tissue obtained from the edge of a glioma will often find it difficult to distinguish between abnormal and normal astrocytes. Abnormal astrocytes can be present in the optic nerve or chiasm without significant enlargement, as evidenced in our patients, where the chiasm looked normal but there was tumour at the site of excision. The most sophisticated imaging techniques currently available are therefore incapable of defining the true edge of a glioma. 
Physiological tests can be used to determine the extent of lesions within the optic pathways. ${ }^{20}$ Visual evoked potentials produced by stimuli in different parts of the visual fields or careful evaluation of the visual fields will demonstrate functional defects produced by tumour. However, quite extensive gliomas often cause only minimal compromise of conduction, since these tests, like the imaging techniques, underestimate the extent of a glioma. This was exemplified by the three patients with an active glioma where tumour was found in the cut end of the optic nerve and assiduous examination of the contralateral visual field and VEP studies failed to demonstrate any abnormality of chiasmal function, though one developed a field defect after 24 months. Lewis et al ${ }^{21}$ CT scanned 217 patients with neurofibromatosis and found tumours of the anterior visual pathways in $15 \%$. Two-thirds of these tumours were entirely unsuspected. Similarly Spencer ${ }^{3}$ reported cases of bilateral thickening of the optic nerves when clinically only one eye was affected. Two of our patients had a similar abnormality.

The difficulties in determining the natural history of these lesions was only partly due to our inability to assess their extent accurately. An even more important factor was our decision about eight years after the start of our study to excise the more rapidly growing and presumably more aggressive tumours.

During the early years of this study all patients were managed as though the gliomas would behave like a hamartoma. All 16 patients in the quiescent group have behaved in this manner, and none has developed evidence of invasion of the chiasm during quite long period of follow-up. Two of the first five patients with a more active course developed a contralateral field defect. One of these patients died during a pneumoencephalogram which was being carried out to determine the size of the chiasm. These events profoundly altered our attitude to the best way of dealing with these tumours. Subsequently additional evidence was provided by one of the patients in whom the optic nerve was excised, with microscopic tumour at the proximal cut end. This patient then developed a progressive field defect and enlargement of the contralateral optic nerve. We believe that these cases show that an optic nerve glioma does have the potential to expand within or extend into the chiasm in a similar manner to the case reported by McDonnell and Miller"2: an 11-year-old boy with an optic nerve glioma initially confined to the orbit and optic canal developed neuroradiological evidence of intracranial extension to involve the hypothalamus and third ventrical.

The results of surgical intervention have been instructive. One patient of the 10 who had a cranio- tomy had an enlarged chiasm, and the optic nerve was not excised. Three of the remainder had tumour at the proximal cut end of the nerve, making it probable that the chiasm was infiltrated by tumour even though it appeared normal to the surgeon. MRI, which was not used in any of these patients, may be capable of detecting small areas of involvement better than a surgeon using an operating microscope. None of our patients with normal MRI studies have had histological evidence of tumour in the chiasm, and two patients who had an enlarged chiasm with normal fields and CT scans were detected with MRI. It will be interesting to see to what extent MRI will enable us to select only those patients with an uninvolved chiasm.

There has been no evidence of tumour growth in either the chiasm or the contralateral optic nerve in any of the six patients where the glioma was totally excised during a follow-up period ranging from 2-10 years. In the three patients who had tumour up to the edge of excision, only one has shown an increase in the size of the chiasm with the development of an enlarged contralateral optic nerve associated with disc oedema and an abnormal field and VEP. The central vision has remained stable at $6 / 6$ over seven years. The other two patients have shown no evidence of spread of their residual tumour over periods of six and nine years. This is in direct contrast to the activity and enlargement of the orbital portion of the tumour prior to craniotomy. Others have noted that incomplete removal of juvenile optic nerve gliomas does not necessarily lead to later clinical recurrence, $, 2:-2=$ and it has been suggested that removal of the main part of the tumour might remove the stimulus to its peripheral growth. ${ }^{26}$ It could be argued that even patients with evidence of chiasmal involvement might benefit from removal of the optic nerve glioma, but we believe this approach is unjustifiable without more definite evidence. Further detailed follow-up of these patients will hopefully provide more evidence about the natural history and effect of treatment on this group of patients.

In reviewing our patients over the last 15 years we have concluded that, while some gliomas are relatively inactive and may well have undergone the deceleration in growth which Alvord et al have suggested, others show a higher level of biological activity, with enlargement and worsening of the clinical picture. One cannot equate this growth of tumour with spread along the optic pathway, but there is sufficient evidence from our study and that of others to indicate that these tumours do pose a threat by direct extension of the astrocytoma into a previously uninvolved chiasm. This threat should be removed by total excision of the involved optic nerve particularly where vision is poor. We do not believe 


\section{Management of Primary Optic Nerve Tumours in the Young}

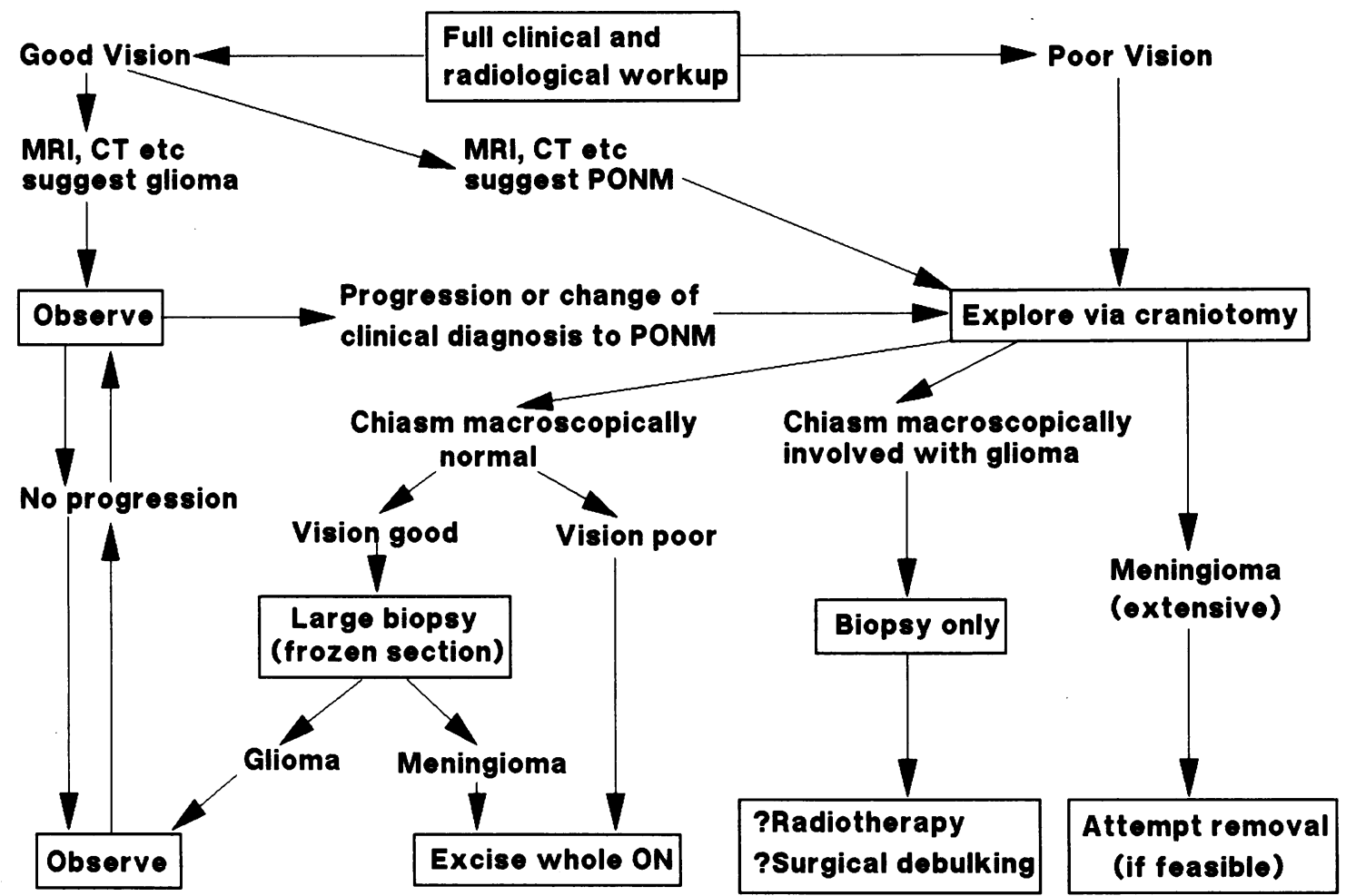

Fig. 4 Flow-chart of recommended management of optic nerve tumours in young patients.

that clinicians who have responsibility for these patients should adopt a totally passive role.

The management of primary optic nerve tumours in young patients without clinical, physiological, or radiological evidence of chiasmal involvement

In another communication ${ }^{8}$ we have drawn attention to the aggressive nature of primary optic nerve meningiomas in young patients. These tumours are capable of invading the middle fossa quite quickly. It is important, therefore, that all primary optic nerve meningiomas which occur under the age of 30 should be diagnosed early and a neurosurgical clearance of the tumour carried out via a transfrontal cranioorbitotomy. In the present communication we have shown that a significant proportion of optic nerve gliomas behave in an active manner and can spread from the nerve to the chiasm and affect the vision in the other eye. Their excision appears to remove this threat. In young patients it is therefore logical to excise all primary optic nerve meningiomas and the active group of optic nerve gliomas, preserving the optic nerve in patients with an indolent or inactive glioma.

In most patients the correct identification of an inactive glioma is not difficult and is based on the typical clinical and radiological appearances. ${ }^{27}$ However, a few patients have features which make the differentiation of meningioma from glioma very difficult even with MRI. It is always possible that the tumour is a meningioma which can spread aggressively (meningioma cases 1 and $2^{*}$ ). This danger can be reduced by excising all optic nerve tumours via a transfrontal approach where vision is poor, a procedure we think justifiable on the grounds that we have seen almost as many patients under the age of 30 with meningioma ${ }^{13}$ as inactive glioma ${ }^{16}$ The pathologist can then decide on the nature of the tumour.

Patients with good vision (6/12 or better)

If MRI and CT scans show an appearance compatible with primary optic nerve meningioma then the patient should be referred for transfrontal neurosurgical exploration. A biopsy should be sent for 
frozen section, and if the diagnosis of meningioma is confirmed then the optic nerve should be completely excised together with the tumour. If it is glioma, the optic nerve should be preserved.

The remainder are patients with either a typical glioma or an optic nerve tumour of doubtful nature. These should be observed to see if there is any change in the size of the tumour, the vision, or the characteristics of the MRI and CT scans which would suggest meningioma or active glioma.

\section{Patients with poor vision (6/18 or worse)}

These patients should have a transfrontal exploration of the chiasm. If despite the normal investigations the chiasm is involved by glioma, further surgery is not indicated. In all other patients the optic nerve should be excised together with the tumour from just anterior to the chiasm to the posterior surface of the globe. If meningioma has spread into the middle fossa, the neurosurgeon will carry out a microsurgical removal of the tumour, if feasible, and possibly treat the area with postoperative radiotherapy. In occassional patients in whom the history suggests the tumour has been present unchanging for a number of years it may be reasonable to continue to observe the patient.

The accompanying flow chart (Fig. 4) indicates the salient points in our management of primary optic nerve tumours in young patients.

Our thanks are due to all the clinicians who referred these patients, Mr Alan Crockard and Professor Lindsay Symon, neurosurgeons at the National Hospitals, Queen Square and Maida Vale, Dr G A S Lloyd and Dr Ivan Moseley, Radiology Department, the staff of the Medical Illustration Department, and Mrs S J Cole, who helped with the typing of the manuscript.

AAMcN was supported by the Royal Australian College of Ophthalmolgists-OPSM travelling fellowship.

\section{References}

1 Hoyt WF, Baghdassarian SA. Optic glioma of childhood. Natural history and rationale for conservative management. Br J Opthalmol 1969; 53: 793-8.

2 Glaser JS, Hoyt WF. Corbett J. Visual morbidity with chiasmal glioma. Arch Ophthalmol 1971; 85: 3-12.

3 Spencer WH. Primary neoplasms of the optic nerve and its sheath; clinical features and current concepts of pathogenetic mechanisms. Trans Am Ophthalmol Soc 1972: 70: 490-528.

4 Wong IG, Lubow M. Management of optic glioma of childhood: a review of 42 cases. Neuro-ophthalmology. St Louis: Mosby, 1972: 6: 51-60.

5 Wright JE, McDonald WI, Call NB. Management of optic nerve gliomas. BrJ Ophthalmol 1980; 64: 545-52.
6 Wong JYC, Uhl V, Wara WM, Sheline GE. Optic gliomas. A reanalysis of the University of California experience. Cancer 1987; 60: 1847-55.

7 Alvord EC, Lofton S. Gliomas of the optic nerve or chiasm Outcome by patients' age, tumor site, and treatment. J Neurosurg 1988; 68: 85-98.

8 Wright JE, McNab AA, McDonald WI. Primary optic nerve sheath meningioma. Br J Ophthalmol 1989; 73: 960-6.

9 Hoyt WF. Meshel LG, Lessell S, Schatz NJ, Suckling RD. Malignant optic glioma of adulthood. Brain 1973: 96: 121-32.

10 Crowe FW, Schull WJ. Diagnostic importance of café-au-lait spot in neurofibromatosis. Arch Intern Med 1953: 91: 758-66.

11 Martin P. Cushing H. Primary gliomas of the chiasm and the optic nerves in their intracranial portion. Arch Ophthalmol 1923: 52; 209-41.

12 Lloyd LA. Gliomas of the optic nerve and chiasm in childhood. Trans Am Ophthalmol Soc 1973; 71 : 488-535.

13 Klug GL. Gliomas of the optic nerve and chiasm in children. Aust NZ J Surg 1977; 47: 596-600.

14 Fletcher WF, Imes RK. Hoyt WF. Chiasmal gliomas: appearance and long-term changes demonstrated by computerised tomography. J Neurosurg 1986; 65: 154-9.

15 Yanoff M. Davis RL. Zimmerman LE. Juvenile pilocytic astrocytoma ('glioma') of the optic nerve. Clinicopathological study of sixty-three cases. In: Jakobiec FA. ed. Ocular and adnexal tumors. Birmingham: Aesculapius. 1978: 685-707.

16 Rush JA, Younge BR, Campbell RJ, MacCarty CS. Optic glioma. Long-term follow-up of 85 histopathologically verified cases. Ophthalmology 1982; 89: 1213-9.

17 Holman RE, Grimson BS, Drayer DP, Buckley EG, Brennan MW. Magnetic resonance imaging in the evaluation of optic gliomas. Am J Ophthalmol 1985: 100: 596-601.

18 Haik BG, Saint Louis L. Bierly J, et al. Magnetic resonance imaging in the evaluation of optic nerve gliomas. Ophthalmology 1987; 94: 709-17.

19 Eidelberg D, Newton MR, Johnson G, et al. Chronic ulilateral optic neuropathy: a magnetic resonance study. Ann Neurol 1988: 24: 3-11.

20 Grosswasser Z, Kriss A. Halliday AM. McDonald WI. Pattern and flash evoked potentials in the assessment and management of optic nerve gliomas. J Neurol Neurosurg Psychiatry 1985; 48: 124-7.

21 Lewis RA. Gerson LP. Axelson KA, Riccardi VM, Whitford RP. von Recklinghausen's neurofibromatosis II. Incidence of optic gliomata. Ophthalmology 1984: 91: 929-35.

22 McDonnell P, Miller NR. Chiasmatic and hypothalamic extension of optic nerve glioma. Arch Ophthalmol 1983; 101: 1412-5.

23 Hudson AC. Primary tumours of the optic nerve. R Lond Ophthalmic Hosp Rev 1912; 18: 317-439.

24 Marquanrdt MD, Zimmerman LE. Histopathology of meningiomas and gliomas of the optic nerve. Hum Pathol 1982: 13: $226-35$.

25 Borit A, Richardson EP. The biological and clinical behaviour of pilocytic astrocytomas of the optic pathways. Brain 1982; 105: $161-87$.

26 Anderson DR, Spencer WH. Ultrastructural and histochemical observations of optic nerve gliomas. Arch Ophthalmol 1970; 83: 324-35.

27 Jacobiec FA, Depot MJ, Kennerdell JS, et al. Combined clinical and computed tomographic diagnosis of orbital glioma and meningioma. Ophthalmology 1984: 91: 137-55.

Accepted.for publication 25 May 1989. 